\title{
Dynamic compression can inhibit chondrogenesis of mesenchymal stem cells
}

${ }^{1}$ Thorpe, S D; ${ }^{1}$ Buckley, C T; ${ }^{1}$ Vinardel, T; ${ }^{1,2} \mathrm{O}$ 'Brien, F J; ${ }^{1,3}$ Campbell, V A; ${ }^{1}$ Kelly, D J

${ }^{1}$ Trinity Centre for Bioengineering, School of Engineering, Trinity College Dublin, Ireland.

${ }^{2}$ Dept. of Anatomy, Royal College of Surgeons in Ireland, Ireland.

${ }^{3}$ Dept. of Physiology, School of Medicine, Trinity College Dublin, Ireland.

Corresponding author:

Dr. Daniel Kelly,

Trinity Centre for Bioengineering,

School of Engineering,

Trinity College Dublin,

Dublin 2,

Ireland.

$\mathrm{Ph}+353-1-8963947 \quad$ Fax $+353-1-6795554$

E-mail: kellyd9@tcd.ie 


\begin{abstract}
The objective of this study was to investigate the influence of dynamic compressive loading on chondrogenesis of mesenchymal stem cells (MSCs) in the presence of TGF- $\beta 3$. Isolated porcine MSCs were suspended in 2\% agarose and subjected to intermittent dynamic compression (10\% strain) for a period of 42 days in a dynamic compression bioreactor. After 42 days in culture, the free-swelling specimens exhibited more intense alcian blue staining for proteoglycans, while immunohistochemical analysis revealed increased collagen type II immunoreactivity. Glycosaminoglycan (GAG) content increased with time for both free-swelling and dynamically loaded constructs, and by day 42 it was significantly higher in both the core $(2.5 \pm 0.21 \% \mathrm{w} / \mathrm{w}$ vs. $0.94 \pm 0.03 \% \mathrm{w} / \mathrm{w})$ and annulus $(1.09 \pm 0.09 \% \mathrm{w} / \mathrm{w}$ vs. $0.59 \pm 0.08 \% \mathrm{w} / \mathrm{w})$ of free-swelling constructs compared to dynamically loaded constructs. This result suggests that further optimization is required in controlling the biomechanical and/or the biochemical environment if such stimuli are to have beneficial effects in generating functional cartilaginous tissue.
\end{abstract}

Keywords: Chondrogenesis; Mesenchymal stem cells; TGF- $\beta 3$; Dynamic compression; Tissue engineering. 


\section{Introduction}

Articular cartilage has a limited capacity for repair. This has motivated the development of cell based therapies such as autologous chondrocyte implantation for the repair of cartilage defects $[1 ; 2]$. A major limiting factor in extending the use of such therapies is obtaining sufficient numbers of differentiated autologous chondrocytes, particularly in elderly and more osteoarthritic (OA) patients. An agerelated loss in chondrogenic capacity has been observed in culture-expanded chondrocytes [3], while collagen synthesis is lower in chondrocytes obtained from OA patients [4]. This may be partially explained by changes in the responsiveness of aged or OA chondrocytes to cytokines and growth factors [5-7]. In addition, OA chondrocytes are likely to have significant DNA damage among other cellular degenerative alterations [8].

Mesenchymal stem cells (MSCs) are a promising alternative cell source for cartilage repair due to both their ease of isolation and expansion, and their chondrogenic differentiation potential [9]. Chondrogenic differentiation of MSCs from different tissue sources has been demonstrated in the presence of growth factors from the transforming growth factor- $\beta$ (TGF- $\beta$ ) superfamily [10-15]. A tissue engineering approach to repair damaged cartilage tissues would involve seeding MSCs into a scaffold and either implanting following minimal in vitro pre-culture, or to culture these constructs for longer periods of time to engineer a more functional cartilaginous tissue. While chondrogenesis does occur in these different systems, it has been demonstrated that the amount of cartilage matrix production and the

subsequent mechanical properties of the tissue is lower with MSCs compared to chondrocytes [16], leading to the suggestion that further optimization may be required 
if MSCs are to be used to engineer cartilaginous tissues with similar functional properties to that obtainable with healthy chondrocytes.

It is well established that the application of appropriate levels of dynamic compressive loading can enhance the biosynthetic activity of chondrocytes [17-23]. It is also believed that the differentiation pathway of MSCs is at least partially regulated by the local mechanical environment. In the absence of chondrogenic growth factors, it has been demonstrated that dynamic compressive loading can enhance chondrogenesis of bone marrow derived MSCs [24-26]. For example, increased aggrecan gene expression has been observed with as few as three 4 hour loading cycles [25], however other studies have observed little dynamic compression induced stimulation of gene expression or matrix synthesis in the absence of chondrogenic growth factors [27]. However, the combined effects of dynamic compression and chondrogenic growth factors (TGF- $\beta$ ) on chondrogenesis of MSCs are generally more complex [24; 27]. Dynamic compression has been shown to up-regulate aggrecan gene expression in the absence of TGF- $\beta$, but to down-regulate it in the presence of TGF- $\beta$ [24]. In both the presence and absence of chondrogenic growth factors, it has been demonstrated that short-term exposure ( $\leq 1$ week) to intermittent dynamic compression leads to enhanced GAG synthesis in the proceeding weeks of culture compared to free swelling controls $[26 ; 28]$. What remains unclear is what influence long-term application of dynamic compression has on the differentiation of MSCs in a chondrogenic environment. This is an important question not only for engineering functional cartilaginous tissues from MSCs, but also to understand how MSCs will respond once implanted into load bearing defects. The goal of this study was to determine how the daily application of dynamic compressive loading influences cartilage specific matrix production by MSCs undergoing chondrogenic 
differentiation in long-term agarose culture. Our initial hypothesis was that dynamic compression would enhance matrix synthesis, leading to increases in the mechanical properties of MSC seeded agarose hydrogels.

\section{Methods}

\section{Cell isolation, expansion and agarose hydrogel encapsulation}

Femora from a 4 month old porcine donor $(\sim 50 \mathrm{~kg})$ were sawn and the gelatinous bone marrow removed under sterile conditions. Porcine MSCs were isolated and expanded according to a modified method developed for human MSCs [29]. Cultures were expanded in high-glucose Dulbecco's Modified Eagle Medium (DMEM GlutaMAX) supplemented with $10 \%$ foetal bovine serum (FBS), and penicillin (100 $\mathrm{U} / \mathrm{mL}$ )-streptomycin (100 $\mu \mathrm{g} / \mathrm{mL})$ (all GIBCO, Biosciences, Ireland). Cultureexpanded MSCs ( $3^{\text {rd }}$ passage) were encapsulated in agarose (Type VII) at $\sim 40^{\circ} \mathrm{C}$, to yield a final gel concentration of $2 \%$ and a cell density of $15 \times 10^{6}$ cells $/ \mathrm{mL}$. The agarose-cell suspension was cast in a stainless steel mould to produce cylindrical discs (Ø $6 \mathrm{~mm} \times 4 \mathrm{~mm}$ thickness). Constructs were maintained in a chemically defined chondrogenic medium (CM) consisting of DMEM GlutaMAX supplemented with penicillin (100 U/mL)-streptomycin (100 $\mu \mathrm{g} / \mathrm{mL})$ (both GIBCO, Biosciences, Ireland), $100 \mu \mathrm{g} / \mathrm{ml}$ sodium pyruvate, $40 \mu \mathrm{g} / \mathrm{ml}$ L-proline, $50 \mu \mathrm{g} / \mathrm{ml}$ L-ascorbic acid-2phosphate, $1 \mathrm{mg} / \mathrm{ml} \mathrm{BSA,} \mathrm{1 \times} \mathrm{insulin-transferrin-selenium,} 100 \mathrm{nM}$ dexamethasone (all from Sigma-Aldrich, Ireland) and $10 \mathrm{ng} / \mathrm{ml}$ recombinant human transforming growth factor- $\beta 3$ (TGF- $\beta 3 ;$ R\&D Systems, UK). Constructs were allowed to equilibrate for 4 days before the addition of dexamethasone and TGF- $\beta 3$, and the initiation of mechanical compressive loading. 


\section{Application of dynamic compression}

Intermittent dynamic compression (DC) was carried out in a custom pneumatic based compressive loading bioreactor with the constructs immersed in CM. The dynamic compression protocol consisted of $\sim 10 \%$ strain amplitude superimposed on a $0.01 \mathrm{~N}$ preload at a frequency of $0.5 \mathrm{~Hz}$. This loading regime was employed for a period of 1 hour, 5 days/week. Free swelling (FS) controls were maintained in the same amount of medium adjacent to the loading device during loading periods. A $50 \%$ medium exchange was performed every 2-3 days.

\section{Mechanical and biochemical analysis}

Constructs were mechanically tested in unconfined compression between impermeable platens using a standard materials testing machine with a $5 \mathrm{~N}$ load cell (Zwick Z005, Roell, Germany). Stress relaxation tests were performed, consisting of a ramp and hold cycle with a ramp displacement of $1 \mu \mathrm{m} / \mathrm{s}$ until $10 \%$ strain was obtained and maintained until equilibrium was reached ( 30 minutes). Dynamic tests were performed immediately after the stress relaxation cycle. A cyclic strain amplitude of $1 \%$ superimposed upon the $10 \%$ strain was applied for 10 cycles at $1 \mathrm{~Hz}$. The compressive equilibrium modulus and dynamic modulus were determined from these tests.

The biochemical content of constructs was assessed at each time point $(0,14$ and 42 days); constructs were cored using a $3 \mathrm{~mm}$ biopsy punch, the wet mass of both annulus and core was recorded and then frozen for subsequent analyses. Annuli and core samples were digested with papain $(125 \mu \mathrm{g} / \mathrm{ml})$ in $0.1 \mathrm{M}$ sodium acetate, $5 \mathrm{mM}$ L-cysteine $\mathrm{HCl}, 0.05 \mathrm{M}$ EDTA, pH 6.0 (all from Sigma-Aldrich, Ireland) at $60^{\circ} \mathrm{C}$ under constant rotation for 18 hours. DNA content was quantified using the Hoechst 
Bisbenzimide 33258 dye assay as described previously [30], with a calf thymus DNA standard. Proteoglycan content was estimated by quantifying the amount of sulphated glycosaminoglycan (GAG) in constructs using the dimethylmethylene blue dyebinding assay (Blyscan, Biocolor Ltd., Northern Ireland), with a chondroitin sulphate standard. Total collagen content was determined by measuring the hydroxyproline content [31], using a hydroxyproline-to-collagen ratio of 1:7.69 [32].

\section{Histology and immunohistochemistry}

At each time point, at least one sample per group was fixed in $4 \%$ paraformaldehyde overnight, rinsed in PBS, and embedded in paraffin. The constructs were embedded such that sectioning at $8 \mu \mathrm{m}$ produced a cross section perpendicular to the disc face. Sections were stained with $1 \%$ alcian blue $8 \mathrm{GX}$ (Sigma-Aldrich, Ireland) in $0.1 \mathrm{M}$ $\mathrm{HCl}$ which stains sGAG, and picro-sirius red to stain collagen. The deposition of collagen type II was indentified by immunohistochemical analysis. A rabbit antihuman polyclonal antibody (concentration $3 \mathrm{~g} / \mathrm{L}$; MD Biosciences, Switzerland) that binds to collagen type II was used. The presence of the collagen type II antibody was subsequently detected by the secondary antibody, biotin-labelled goat anti-rabbit polyclonal (concentration $1 \mathrm{~g} / \mathrm{L}$; Sigma-Aldrich, Ireland). Negative and positive controls, trachea and cartilage respectively, were included.

\section{Statistical Analysis}

Statistical analyses were preformed using GraphPad Prism (Version 4.03) software with 3-4 samples analysed for each experimental group. Two-way ANOVA was used for analysis of variance with Bonferroni post-tests to compare between groups. 
Numerical and graphical results are displayed as mean \pm standard deviation. Significance was accepted at a level of $p<0.05$.

\section{Results}

There were no statistical differences in the DNA content between the FS and DC groups at any time point; or between core and annular regions within any group of constructs (Fig. 1A).

The GAG content was significantly higher after both 14 and 42 days in culture compared to the corresponding samples on day 0 (Fig. 1B). No difference was found between the FS and DC groups for annulus and core respectively at day 14. However at day 42 , both annulus $(1.09 \pm 0.09 \% \mathrm{w} / \mathrm{w}$ vs. $0.59 \pm 0.08 \% \mathrm{w} / \mathrm{w})$ and core $(2.5 \pm 0.21$ $\% \mathrm{w} / \mathrm{w}$ vs. $0.94 \pm 0.03 \% \mathrm{w} / \mathrm{w})$ regions for FS constructs were significantly greater $(p<0.001)$ than DC constructs. GAG content in the core region of all construct groups was significantly greater than that in the annulus (Fig. 1B). Collagen content was also higher in FS constructs compared to DC constructs at day 42 (Fig. 1C).

Staining of construct sections with alcian blue for sulphated proteoglycan revealed a steady accretion of positive staining for both FS and DC conditions (Fig. 2). At both days 14 and 42 the FS constructs exhibited more intense positive staining for GAG than the DC constructs. Similar trends were seen in the Picro-sirius staining for collagen (data not shown). This staining corresponded to biochemical results, with a less intense staining around the periphery of the construct. The collagen type II immunohistochemistry (Fig. 2) provides evidence of chondrogenic differentiation, again showing stronger staining away from the construct edge. The DC constructs exhibit a more homogeneous spatial staining in each case than the FS constructs. 
The equilibrium and dynamic mechanical properties were dependent on time in culture $(p<0.001)$ and mechanical stimulation $(p<0.001)$ (Fig.3). However, significant differences between FS and DC were only seen at day $42(p<0.001)$; the equilibrium modulus of day $42 \mathrm{FS}$ constructs reached $49.9 \pm 0.6 \mathrm{kPa}$ compared to $24.4 \pm 1.5 \mathrm{kPa}$ for the DC constructs. The $1 \mathrm{~Hz}$ dynamic modulus followed a similar trend with day $42 \mathrm{FS}$ constructs reaching $230.7 \pm 3.4 \mathrm{kPa}$ compared to $111.4 \pm 4.1 \mathrm{kPa}$ for the DC constructs. Notably, the mechanical properties of DC constructs did not increase between day 14 and 42.

\section{Discussion}

Whether injected directly in-vivo, or cultured ex-vivo on scaffolds prior to implantation, understanding the response of MSCs to physical forces is of critical importance when developing cell based therapies for cartilage repair. Our hypothesis was that intermittent dynamic compression applied daily to MSCs in agarose culture in a chemically defined medium supplemented with TGF- $\beta 3$ would enhance chondrogenesis, resulting in the formation of a more functional cartilaginous tissue. No evidence was found to support this hypothesis. Rather dynamic compressive loading was observed to inhibit chondrogenesis of MSCs in the presence of TGF- $\beta 3$.

There are a number of factors that could explain the observed results. Our initial hypothesis was based on the observation that dynamic compressive loading of chondrocytes in agarose culture stimulates cartilage specific gene expression and protein synthesis $[20 ; 23]$. In this study, dynamic compressive loading was applied shortly after the MSCs had been exposed to chondrogenic growth factors. At this early stage in the differentiation process, it is perhaps not surprising that MSCs do not respond to biophysical stimulation in a similar manner to fully differentiated articular 
chondrocytes. Mouw et al. [27] observed that the response of bone marrow derived MSCs in agarose culture to dynamic compression varied during the process of chondrogenesis, with dynamic compression only enhancing chondrogenesis following 16 days of pre-culture in free swelling conditions in the presence of TGF- $\beta 1$. It was suggested that the developing pericellular and extracellular matrix may be regulating mechano-stimulation of MSCs undergoing chondrogenesis. Cartilage matrix synthesis by articular chondrocytes in dynamically compressed agarose gels is greater at later time points than earlier time points [20], implying that a well developed matrix may be required for the transduction of mechanical compression to a cellular biosynthetic response.

Another possible explanation is that the type, magnitude, frequency or duration of the applied mechanical stimulus in this study (unconfined compression at $10 \%$ strain at $0.5 \mathrm{~Hz}$ for 1 hour/day) inhibits chondrogenesis of MSCs. The application of this loading protocol produces a complex mechanical environment within the agarose hydrogel, with magnitudes of deformation, hydrostatic pressure and fluid velocities that vary spatially and temporally. Theoretical models have suggested that the mechanical environment can regulate the differentiation pathway of MSCs [33; 34], and it is possible that excessive levels of deformation or fluid flow in the agarose hydrogel, particularly in the absence of a fully developed pericellular matrix to regulate mechano-transduction, could be promoting the formation of a more fibroblastic phenotype. Immunohistochemical analysis also revealed the presence of type I collagen in both FS and DC constructs (data not shown). Comparisons of different bioreactor systems for cartilage tissue engineering suggest that fluid flow plays a role in regulating chondrogenesis [35]. Chondrocyte differentiation has also been shown to be modulated by the frequency and duration of cyclic compressive 
loading [36]. Altering parameters of the dynamic compression loading protocol such as the strain magnitude and frequency would allow this hypothesis to be investigated further.

These factors do not explain the results of similar studies where mechanical loading in the absence of TGF- $\beta$ enhances chondrogenesis [24-26], even when applied shortly after agarose encapsulation. This would suggest that a more complex interplay exists between the biophysical and biochemical environment in regulating chondrogenesis of MSCs, leading to the inhibition of cartilage-specific matrix production observed in this study. There are many potential interactions that need to be considered. In free swelling cultures, it was observed that total GAG synthesis was greater in the core compared to the annulus of the agarose hydrogels (Fig. 1). This may be partially explained by diffusion of GAGs from the annulus of the construct into the surrounding media. Anther explanation may be diffusion limitations or cell consumption at the periphery of the hydrogel producing a radial gradient in the molecules responsible for regulating chondrogenesis. For example, lower concentrations of TGF- $\beta 3$ or oxygen towards the core may be promoting chondrogenesis. If this is the case, the enhanced transport and concentration of these molecules due to dynamic compression [37] may explain the inhibition of chondrogenesis in this study. For example, hypoxia has been shown to promote chondrogenesis of MSCs in the presence of chondrogenic growth factors [38], however it has also been shown that diffusional limitations in oxygen supply can result in more anaerobic conditions resulting in markedly reduced rates in the growth of chondrocyte seeded polymer scaffolds [39]. The biosynthetic response of cartilage explants to TGF- $\beta$ treatment has been shown to be dose dependant, reaching saturation at 10ng/ml [40]. Chondrogenic differentiation of MSCs would also appear 
to depend on the dose of TGF- $\beta$, with $5 \mathrm{ng} / \mathrm{ml}$ of TGF- $\beta 1$ reported as optimal in monolayer culture [41]. In pellet culture it has been reported that at a concentration of $1 \mathrm{ng} / \mathrm{ml}$ of TGF- $\beta 1$, chondrogenesis is limited to the outer third of the cell aggregate, with more homogenous chondrogenesis at $10 \mathrm{ng} / \mathrm{ml}$ [11]. In addition, stimulation of the TGF- $\beta$ signalling pathway could modulate mechano-transduction, or alternatively, mechanical stimulation may modulate TGF- $\beta$ signalling [27]. Interestingly, dynamic compressive loading of chondrocyte seeded agarose hydrogels applied concurrently with exposure to $10 \mathrm{ng} / \mathrm{ml}$ of TGF- $\beta 3$ has also been shown to inhibit GAG synthesis [42]. In the same study [42], deformational loading initiated after culturing for two weeks in the presence of TGF- $\beta 3$ yielded significantly stiffer constructs than freeswelling controls. Adopting a similar loading protocol with MSCs undergoing chondrogenesis may produce a more anabolic response to dynamic deformational loading. The results of these studies have important implications for post-operative rehabilitation regimes following cell-based therapies to repair cartilage, and for designing scaffolds to control the biochemical and biophysical environment of MSCs following implantation.

\section{Acknowledgements}

Funding was provided by Science Foundation Ireland (07-RFP- ENMF142) and Enterprise Ireland (PC / 2006/ 384). 


\section{References}

[1] M. Brittberg, A. Lindahl, A. Nilsson, C. Ohlsson, O. Isaksson, and L. Peterson, Treatment of deep cartilage defects in the knee with autologous chondrocyte transplantation. N Engl J Med 331 (1994) 889-95.

[2] L. Peterson, T. Minas, M. Brittberg, A. Nilsson, E. Sjogren-Jansson, and A. Lindahl, Two- to 9-year outcome after autologous chondrocyte transplantation of the knee. Clin Orthop Relat Res (2000) 212-34.

[3] A. Barbero, S. Grogan, D. Schafer, M. Heberer, P. Mainil-Varlet, and I. Martin, Age related changes in human articular chondrocyte yield, proliferation and post-expansion chondrogenic capacity. Osteoarthritis Cartilage 12 (2004) 47684.

[4] T. Tallheden, C. Bengtsson, C. Brantsing, E. Sjogren-Jansson, L. Carlsson, L. Peterson, M. Brittberg, and A. Lindahl, Proliferation and differentiation potential of chondrocytes from osteoarthritic patients. Arthritis Res Ther 7 (2005) R560-8.

[5] Z. Fan, B. Bau, H. Yang, S. Soeder, and T. Aigner, Freshly isolated osteoarthritic chondrocytes are catabolically more active than normal chondrocytes, but less responsive to catabolic stimulation with interleukin-1beta. Arthritis Rheum 52 (2005) 136-43.

[6] Z. Fan, S. Chubinskaya, D.C. Rueger, B. Bau, J. Haag, and T. Aigner, Regulation of anabolic and catabolic gene expression in normal and osteoarthritic adult human articular chondrocytes by osteogenic protein-1. Clin Exp Rheumatol 22 (2004) 103-6.

[7] J.A. Martin, S.M. Ellerbroek, and J.A. Buckwalter, Age-related decline in chondrocyte response to insulin-like growth factor-I: the role of growth factor binding proteins. J Orthop Res 15 (1997) 491-8.

[8] T. Aigner, J. Haag, J. Martin, and J. Buckwalter, Osteoarthritis: aging of matrix and cells--going for a remedy. Curr Drug Targets 8 (2007) 325-31.

[9] A.I. Caplan, Mesenchymal stem cells. J Orthop Res 9 (1991) 641-50.

[10] M. Iwasaki, K. Nakata, H. Nakahara, T. Nakase, T. Kimura, K. Kimata, A.I. Caplan, and K. Ono, Transforming growth factor-beta 1 stimulates chondrogenesis and inhibits osteogenesis in high density culture of periosteum-derived cells. Endocrinology 132 (1993) 1603-8.

[11] B. Johnstone, T.M. Hering, A.I. Caplan, V.M. Goldberg, and J.U. Yoo, In vitro chondrogenesis of bone marrow-derived mesenchymal progenitor cells. Exp Cell Res 238 (1998) 265-72.

[12] A.M. Mackay, S.C. Beck, J.M. Murphy, F.P. Barry, C.O. Chichester, and M.F. Pittenger, Chondrogenic differentiation of cultured human mesenchymal stem cells from marrow. Tissue Eng 4 (1998) 415-28.

[13] K. Nishimura, L.A. Solchaga, A.I. Caplan, J.U. Yoo, V.M. Goldberg, and B. Johnstone, Chondroprogenitor cells of synovial tissue. Arthritis Rheum 42 (1999) 2631-7.

[14] J.U. Yoo, T.S. Barthel, K. Nishimura, L. Solchaga, A.I. Caplan, V.M. Goldberg, and B. Johnstone, The chondrogenic potential of human bone-marrow-derived mesenchymal progenitor cells. J Bone Joint Surg Am 80 (1998) 1745-57.

[15] P.A. Zuk, M. Zhu, H. Mizuno, J. Huang, J.W. Futrell, A.J. Katz, P. Benhaim, H.P. Lorenz, and M.H. Hedrick, Multilineage cells from human adipose tissue: implications for cell-based therapies. Tissue Eng 7 (2001) 211-28. 
[16] R.L. Mauck, X. Yuan, and R.S. Tuan, Chondrogenic differentiation and functional maturation of bovine mesenchymal stem cells in long-term agarose culture. Osteoarthritis Cartilage 14 (2006) 179-89.

[17] Y.J. Kim, R.L. Sah, A.J. Grodzinsky, A.H. Plaas, and J.D. Sandy, Mechanical regulation of cartilage biosynthetic behavior: physical stimuli. Arch Biochem Biophys 311 (1994) 1-12.

[18] M.J. Palmoski, and K.D. Brandt, Effects of static and cyclic compressive loading on articular cartilage plugs in vitro. Arthritis Rheum 27 (1984) 675-81.

[19] R.L. Sah, Y.J. Kim, J.Y. Doong, A.J. Grodzinsky, A.H. Plaas, and J.D. Sandy, Biosynthetic response of cartilage explants to dynamic compression. J Orthop Res 7 (1989) 619-36.

[20] M.D. Buschmann, Y.A. Gluzband, A.J. Grodzinsky, and E.B. Hunziker, Mechanical compression modulates matrix biosynthesis in chondrocyte/agarose culture. J Cell Sci 108 ( Pt 4) (1995) 1497-508.

[21] T. Davisson, S. Kunig, A. Chen, R. Sah, and A. Ratcliffe, Static and dynamic compression modulate matrix metabolism in tissue engineered cartilage. $\mathrm{J}$ Orthop Res 20 (2002) 842-8.

[22] R.L. Mauck, S.L. Seyhan, G.A. Ateshian, and C.T. Hung, Influence of seeding density and dynamic deformational loading on the developing structure/function relationships of chondrocyte-seeded agarose hydrogels. Ann Biomed Eng 30 (2002) 1046-56.

[23] R.L. Mauck, M.A. Soltz, C.C. Wang, D.D. Wong, P.H. Chao, W.B. Valhmu, C.T. Hung, and G.A. Ateshian, Functional tissue engineering of articular cartilage through dynamic loading of chondrocyte-seeded agarose gels. J Biomech Eng 122 (2000) 252-60.

[24] J.J. Campbell, D.A. Lee, and D.L. Bader, Dynamic compressive strain influences chondrogenic gene expression in human mesenchymal stem cells. Biorheology 43 (2006) 455-70.

[25] C.Y. Huang, K.L. Hagar, L.E. Frost, Y. Sun, and H.S. Cheung, Effects of cyclic compressive loading on chondrogenesis of rabbit bone-marrow derived mesenchymal stem cells. Stem Cells 22 (2004) 313-23.

[26] R.L. Mauck, B.A. Byers, X. Yuan, and R.S. Tuan, Regulation of cartilaginous ECM gene transcription by chondrocytes and MSCs in 3D culture in response to dynamic loading. Biomech Model Mechanobiol 6 (2007) 113-25.

[27] J.K. Mouw, J.T. Connelly, C.G. Wilson, K.E. Michael, and M.E. Levenston, Dynamic compression regulates the expression and synthesis of chondrocytespecific matrix molecules in bone marrow stromal cells. Stem Cells 25 (2007) 655-63.

[28] P. Angele, D. Schumann, M. Angele, B. Kinner, C. Englert, R. Hente, B. Fuchtmeier, M. Nerlich, C. Neumann, and R. Kujat, Cyclic, mechanical compression enhances chondrogenesis of mesenchymal progenitor cells in tissue engineering scaffolds. Biorheology 41 (2004) 335-46.

[29] D.P. Lennon, and A.I. Caplan, Isolation of human marrow-derived mesenchymal stem cells. Exp Hematol 34 (2006) 1604-5.

[30] Y.J. Kim, R.L. Sah, J.Y. Doong, and A.J. Grodzinsky, Fluorometric assay of DNA in cartilage explants using Hoechst 33258. Anal Biochem 174 (1988) 168-76.

[31] W. Kafienah, and T.J. Sims, Biochemical methods for the analysis of tissueengineered cartilage. Methods Mol Biol 238 (2004) 217-30. 
[32] N.Y. Ignat'eva, N.A. Danilov, S.V. Averkiev, M.V. Obrezkova, V.V. Lunin, and E.N. Sobol, Determination of hydroxyproline in tissues and the evaluation of the collagen content of the tissues. J Anal Chem 62 (2007) 51-57.

[33] D.J. Kelly, and P.J. Prendergast, Mechano-regulation of stem cell differentiation and tissue regeneration in osteochondral defects. Journal of Biomechanics 38 (2005) 1413-1422.

[34] P.J. Prendergast, R. Huiskes, and K. Søballe, Biophysical stimuli on cells during tissue differentiation at implant interfaces. Journal of Biomechanics 30 (1997) 539-548.

[35] G. Vunjak-Novakovic, I. Martin, B. Obradovic, S. Treppo, A.J. Grodzinsky, R. Langer, and L.E. Freed, Bioreactor cultivation conditions modulate the composition and mechanical properties of tissue-engineered cartilage. J Orthop Res 17 (1999) 130-8.

[36] S.H. Elder, S.A. Goldstein, J.H. Kimura, L.J. Soslowsky, and D.M. Spengler, Chondrocyte differentiation is modulated by frequency and duration of cyclic compressive loading. Ann Biomed Eng 29 (2001) 476-82.

[37] R.L. Mauck, C.T. Hung, and G.A. Ateshian, Modeling of neutral solute transport in a dynamically loaded porous permeable gel: implications for articular cartilage biosynthesis and tissue engineering. J Biomech Eng 125 (2003) 60214.

[38] M. Kanichai, D. Ferguson, P.J. Prendergast, and V.A. Campbell, Hypoxia promotes chondrogenesis in rat mesenchymal stem cells: a role for AKT and hypoxia-inducible factor (HIF)-1alpha. J Cell Physiol 216 (2008) 708-15.

[39] B. Obradovic, R.L. Carrier, G. Vunjak-Novakovic, and L.E. Freed, Gas exchange is essential for bioreactor cultivation of tissue engineered cartilage. Biotechnol Bioeng 63 (1999) 197-205.

[40] T.I. Morales, and A.B. Roberts, Transforming growth factor beta regulates the metabolism of proteoglycans in bovine cartilage organ cultures. J Biol Chem 263 (1988) 12828-31.

[41] A.A. Worster, B.D. Brower-Toland, L.A. Fortier, S.J. Bent, J. Williams, and A.J. Nixon, Chondrocytic differentiation of mesenchymal stem cells sequentially exposed to transforming growth factor-beta1 in monolayer and insulin-like growth factor-I in a three-dimensional matrix. J Orthop Res 19 (2001) 738-49.

[42] E.G. Lima, L. Bian, K.W. Ng, R.L. Mauck, B.A. Byers, R.S. Tuan, G.A. Ateshian, and C.T. Hung, The beneficial effect of delayed compressive loading on tissue-engineered cartilage constructs cultured with TGF-beta3. Osteoarthritis Cartilage 15 (2007) 1025-33. 


\section{Figure Legends}

Fig. 1. Biochemical composition of both core and annular regions for FS and DC groups; (A): DNA content (ng DNA/mg construct w/w), (B): GAG content (\% construct $\mathrm{w} / \mathrm{w}),(\mathrm{C})$ : Collagen content $(\%$ construct $\mathrm{w} / \mathrm{w}) . * p<0.05$, **p<0.01, ***p<0.001. ! indicates significant difference $(\mathrm{p}<0.001)$ between FS and DC constructs (both core and annulus).

Fig. 2. Representative alcian blue staining (top) and collagen type II immunohistochemistry (bottom) at day 0 and day 42 for both FS and DC construct groups. Scale bar: $1 \mathrm{~mm}$.

Fig. 3. Equilibrium compressive modulus (left) and $1 \mathrm{~Hz}$ dynamic modulus (right) of both FS and DC groups at each time point. $* * * p<0.001$. 

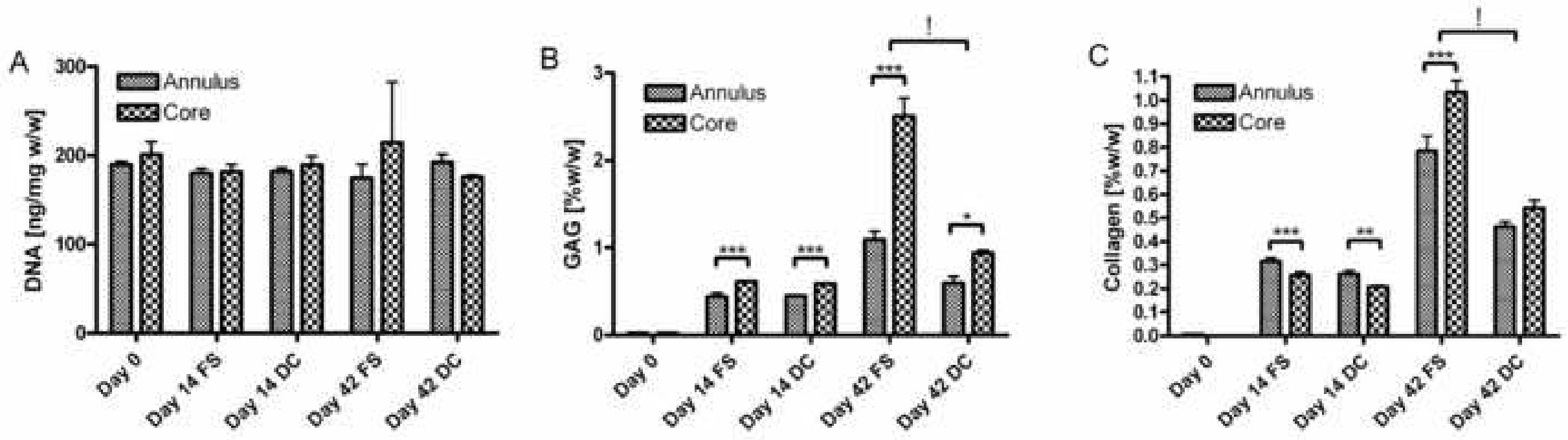

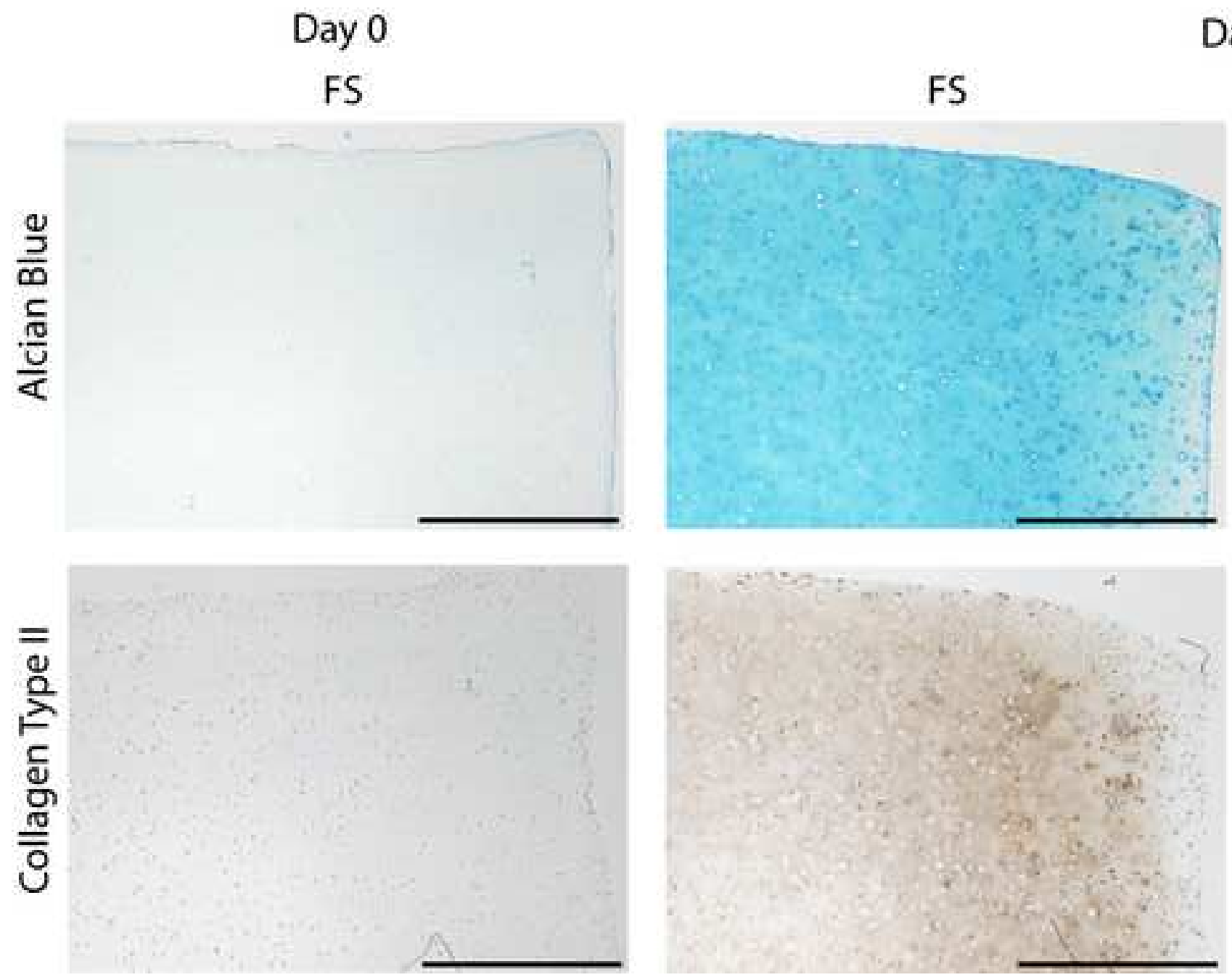

Day 42

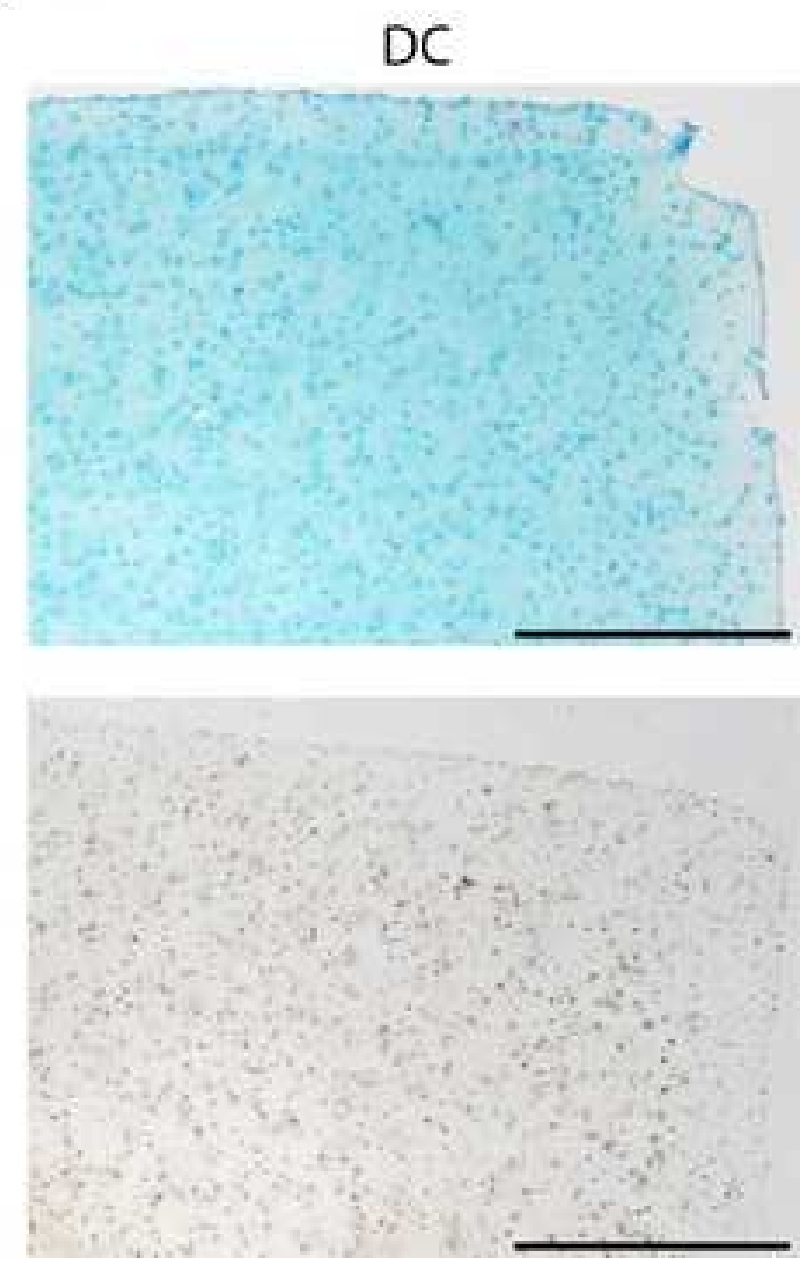




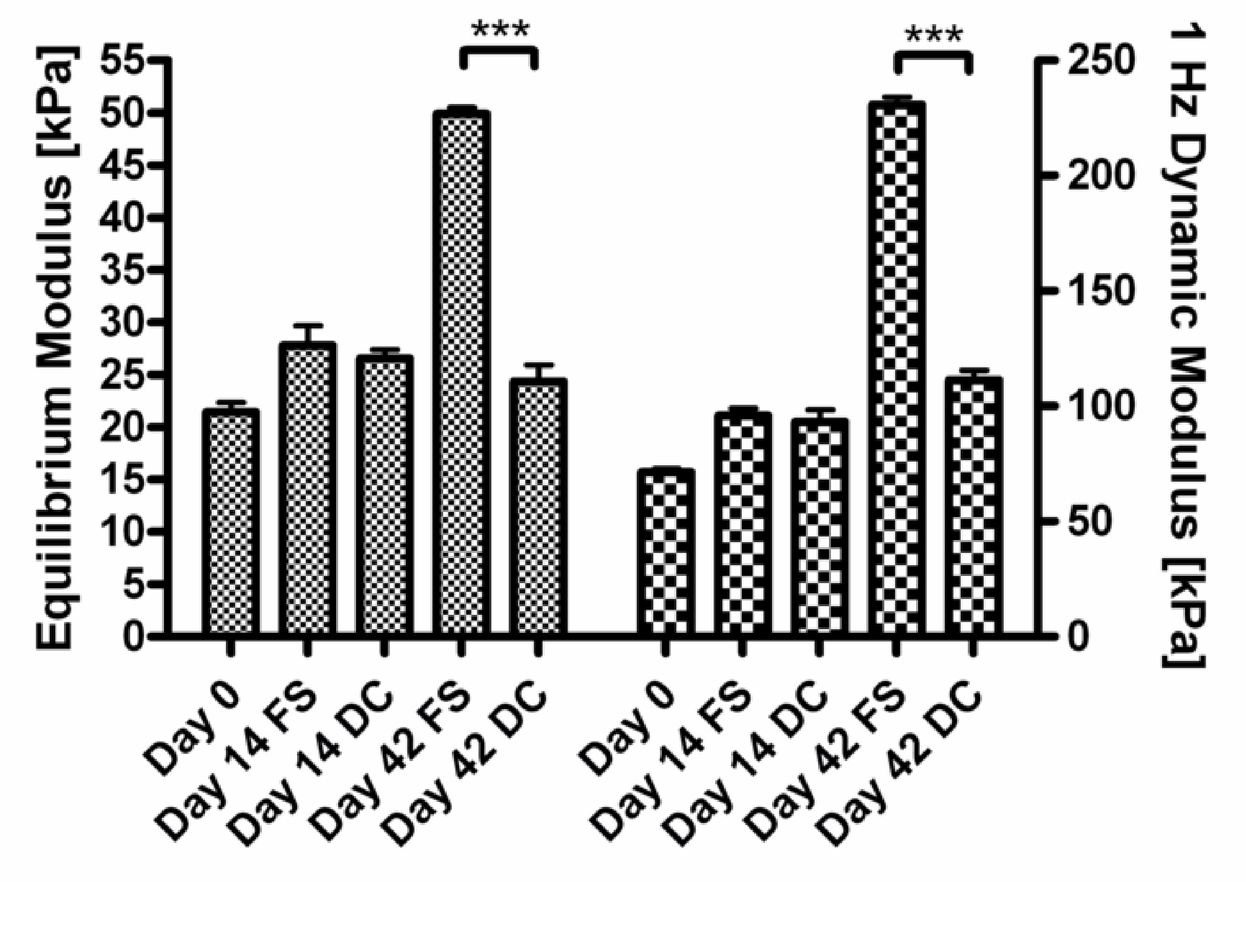

cher here

.

